\title{
NPS2390, a selective calcium-sensing receptor antagonist controls the phenotypic modulation of hypoxic human pulmonary arterial smooth muscle cells by regulating autophagy
}

\author{
Xue Peng ${ }^{1,2}$, Can Wei ${ }^{1}$, Hong-Zhu Li ${ }^{1}$, Hong-Xia Li $^{1}$, Shu-Zhi Bai ${ }^{1}$, Li-Na Wang ${ }^{1}$, \\ Yu-Hui Xi ${ }^{1}$, Jin Yan $^{3}$, Chang-Qing $\mathrm{Xu}^{1,4}$ * \\ 'Department of Pathophysiology, Harbin Medical University, Harbin, Heilongjiang Province, China; \\ ${ }^{2}$ Department of Forensic Medicine, Harbin Medical University, Harbin, Heilongjiang Province, China; \\ ${ }^{3}$ Laboratory of Medical Genetics, Harbin Medical University, Harbin, Heilongjiang Province, China; \\ ${ }^{4}$ Bio-pharmaceutical Key Laboratory of Heilongjiang Province, Harbin, Heilongjiang Province, China
}

\section{ABSTRACT}

Background and Objectives: Calcium-sensing receptor (CaSR) is known to regulate hypoxia-induced pulmonary hypertension $(\mathrm{HPH})$ and vascular remodeling via the phenotypic modulation of pulmonary arterial smooth muscle cells (PASMCs) in small pulmonary arteries. Moreover, autophagy is an essential modulator of VSMC phenotype. But it is not clear whether CaSR can regulate autophagy involving the phenotypic modulation under hypoxia. Methods: The viability of human PASMCs was detected by cell cycle and BrdU. The expressions of proliferation protein, phenotypic marker protein, and autophagy protein in human PASMCs were determined by western blot. Results: Our results showed that hypoxia-induced autophagy was considerable at $24 \mathrm{~h}$. The addition of NPS2390 decreased the expression of autophagy protein and synthetic phenotype marker protein osteopontin and increased the expression of contractile phenotype marker protein SMA- $a$ and calponin via suppressing downstream PI3K/ Akt/mTOR signal pathways. Conclusions: Our study demonstrates that treatment of NPS2390 was conducive to inhibit the proliferation and reverse phenotypic modulation of PASMCs by regulating autophagy levels.

Key words: NPS2390, calcium-sensing receptors, hypoxia, pulmonary arterial smooth muscle cells, phenotypic modulation, autophagy

\section{INTRODUCTION}

The hypoxic pulmonary hypertension $(\mathrm{HPAH})$ is a clinical syndrome caused from increasing pulmonary vascular resistance, which can cause serious impairment of heart and lung function. Hypoxic pulmonary vascular remodeling (HPVR) is an important pathologic basis, but the exact mechanisms have not been fully elucidated. The abnormal proliferation of pulmonary arterial smooth muscle cells (PASMCs) play an important role during this process. Our previous study have found that phenotypic modulation of PASMCs is a key step in initiating HPVR, but the molecular mechanisms controlling the phenotypic modulation of PASMCs are still not fully understood.

Autophagy is a process of phagocytizing its own cytoplasmic proteins or organelles, coating them into vesicles, and fusing with lysosomes to form autophagosomes, which degrade the contents of their entrapment. ${ }^{[1]}$ LC3 is an widely accepted autophagic marker. When autophagy is formed, cytosolic LC3 (LC3-I) will enzymatically cleave a small segment of the polypeptide and transform it into an autophagosome membrane type (LC3-II)..$^{[2,3]}$ The previous study has shown that autophagic protein LC3B play a protective role during the development of PAH through regulating 
hypoxic-induced cell proliferation. ${ }^{[4]}$ Moreover, inhibition of autophagy by drugs can inhibit PDGF on synthetic phenotype. ${ }^{[5,6]}$ Autophagy may participate in the process of phenotypic modulation of VSMCs. Furthermore, the molecular mechanism of autophagy is complicated and related to several distinct signaling pathways. Especially, the $\mathrm{PI} 3 \mathrm{~K} / \mathrm{Akt} / \mathrm{m}$ TOR signaling pathways were demonstrated to regulate autophagy under certain conditions. ${ }^{[7,8]}$ However, the role of autophagy was still not fully understood in hypoxic pulmonary hypertension (HPAH).

Calcium-sensing receptor (CaSR) is a G-protein-coupled receptor; its activation can increase the concentration of intracellular calcium. In the previous study, we have verified that the activation of CaSR is related to hypoxia-induced proliferation of rat PASMCs through MEK1/ERK1,2 and PI3K pathways. Recently, we also discovered that the expression of CaSR was increased and involved in the phenotypic modulation of PASMCs in small pulmonary artery and vascular remodeling in hypoxic rat. Besides, $\left[\mathrm{Ca}^{2+}\right]_{\mathrm{i}}$ plays an important role in the process of autophagy. ${ }^{[9,10]}$ However, whether $\mathrm{CaSR}$ regulates the phenotypic modulation via mediating autophagy in human PASMCs under hypoxia is still unclear.

In our study, we observed whether CaSR antagonist can regulate proliferation and phenotypic modulation of PASMCs under hypoxia and whether PI3K/Akt/mTOR signaling pathway is related to these processes.

\section{MATERIAL AND METHODS}

\section{Cell culture}

Human pulmonary artery smooth muscle cells (HPASMCs) and medium SMCM was purchased from American ScienCell Company. HPASMCs cells were cultured in SMCM in complete medium $(89 \% \mathrm{DMEM}+10 \%$ fetal calf Serum $+1 \%$ growth factor), and $100 \mathrm{U} / \mathrm{mL}$ was added to the medium Cyan-streptomycin. The cells were placed at $37^{\circ} \mathrm{C}, 21 \% \mathrm{O}_{2}, 5 \% \mathrm{CO}_{2}, 74 \%$ subculture in $\mathrm{N}_{2}$, incubator under saturated humidity, take within 6 generations counting cells in experiments.

\section{Hypoxia treatment of HPASMCs}

The original medium of HPASMCs was discarded and 1\% fetal bovine serum was added to DMEM. The culture was incubated for $12 \mathrm{~h}$ for synchronization and then changed to SMCM culture. The cells were placed in a hypoxic incubator and treated at $1 \% \mathrm{O}_{2}$ concentration.

\section{BrdU incorporation and detection}

$\mathrm{BrdU}$ was incorporated into PASMCs, and proliferation of different treatment groups was detected using immunofluorescence technique. Cells from different treatment groups were cultured in 96-well plates for 44 $\mathrm{h}$, and then BrdU was added to these cells and continued culturing for $4 \mathrm{~h}$ at $38.5^{\circ} \mathrm{C}$. Subsequently, the cells were fixed with $4 \%$ paraformaldehyde for $20 \mathrm{~min}$, washed for 3 times with phosphate buffered saline with Tween-20 (PBST) at a concentration of $2 \mathrm{~mol} / \mathrm{L} \mathrm{HCl}, 37^{\circ} \mathrm{C}$ and denatured for $30 \mathrm{~min}$. After adding $0.1 \mathrm{~mol} / \mathrm{L}$ of sodium tetraborate, the cells were kept for $5 \mathrm{~min}$ and washed with PBST for 3 times; then the cells were blocked with readyto-use goat serum for $20 \mathrm{~min}$ at room temperature. After adding BrdU primary antibody G3G4 (1:200), the cells were incubated overnight at $4{ }^{\circ} \mathrm{C}$ and washed for 3 times with PBST. TRIFC-labeled goat anti-mouse secondary antibody (1:500) was added to the cells and incubated for $1 \mathrm{~h}$ at $37^{\circ} \mathrm{C}$ and washed for 3 times with PBST after incubation. Finally, DAPI was added and the cells were kept at room temperature for $30 \mathrm{~min}$, after which the cells were washed for 3 times with PBST. The BrdU incorporation index was calculated by BrdU-labeled nuclei and DAPI-stained nuclei ratio under $I \times 70$ fluorescence microscopy.

\section{Cell cycle assays}

For cell cycle analysis, the PASMCs to be tested were washed with a phosphate buffered saline solution, placed in a centrifuge tube, and centrifuged at 1,000 rpm for 5 min. The supernatant was discarded, and the cells were resuspended in $80 \%$ ethanol at $4{ }^{\circ} \mathrm{C}$. The cells were centrifuged again for $24 \mathrm{~min}$ at 1,000 rpm for $24 \mathrm{~min}$. The fixative was discarded, and the cells were resuspended in propidium iodide. Cell cycle analysis was performed with a Cy-toFLEX S flow cytometer (manufactured by Beckman Coulter) after $30 \mathrm{~min}$ at room temperature.

\section{Western blot}

The cells were collected and $300 \mu \mathrm{L}$ of protein lysate was added. The cells were placed on ice for $15 \mathrm{~min}$ and then centrifuged at $15{ }^{\circ} \mathrm{C} / 12,000 \mathrm{rpm}$ for $15 \mathrm{~min}$ The supernatant was collected. After adjusting the concentration, the protein was boiled in boiling water for $10 \mathrm{~min}$. SDS-PAGE gels were prepared and filled with $40 \mu \mathrm{g}$ of sample per well for electrophoresis. After 300$\mathrm{mA}$ wet rotation for $120 \mathrm{~min}$, the PVDF membrane was blocked at room temperature for $1 \mathrm{~h}$ and then added to the antibody dilution of CaSR (1:800), calponin (1:500), SMA- $\alpha$ (1:1000), OPN (1:500), PCNA (1:1000), Ki67(1:1000), LC3 (1:1000), caspase-3(1:1000), AKT (1:1000), p-AKT (1:1000), PI3K (1:1000), p-PI3K (1:1000), mTOR (1:1000), p-mTOR $(1: 1000)$, and GAPDH $(1: 1000)$ at $4{ }^{\circ} \mathrm{C}$ overnight. The gel was wash with TBST for 3 times, each time for $10 \mathrm{~min}$, and then horseradish-labeled goat anti-rabbit secondary antibody (1: 50,000) was added, The gel was incubate for $1 \mathrm{~h}$ at room temperature and washed with TBST for 3 times; each protein expression was detected by ECL chemiluminescence at $10 \mathrm{~min}$. The resulting protein 
strip image, using Quality one software to analyze the image to GAPDH light density. The degree value is used as an internal parameter to correct the optical density value of the target protein.

\section{Measurement of intracellular calcium in HPASMCS}

Fluo-4 AM calcium ion fluorescent probe was used to detect the intracellular calcium ion density in each group. The cell density was $2 \times 10^{5} / \mathrm{mL}$. The laser confocal small dish was inserted. After treating the cells according to the above group, $10 \mu \mathrm{mol} / \mathrm{L}$ of Fluo- $4 \mathrm{AM}$ was added according to the instructions. The cells were then incubated in the dark for $30 \mathrm{~min}$, after which they were rinsed in PBS with calcium-free buffer repeatedly, centrifuged for 2 times, and observed under laser confocal microscope.

\section{Statistics}

Data were analyzed using SPSS21.0 statistical software, all results according to the mean \pm SEM, the comparison between the different groups was tested in One-way ANOVA, $P<0.05$ was considered statistically significant.

\section{RESULTS}

\section{Hypoxia increases the expression of CaSR in cultured HPASMCS}

Primary cultured HPASMCs were incubated for different times $(12,24$, and $48 \mathrm{~h})$ at $1 \%$ oxygen concentration in the hypoxia chamber, the control group was incubated under normoxia. Western blot analysis indicated that the CaSR expression in HPASMCs under hypoxia was increased from $12 \mathrm{~h}$ and peaked at $24 \mathrm{~h}(P<0.05$, Figure 1$)$.

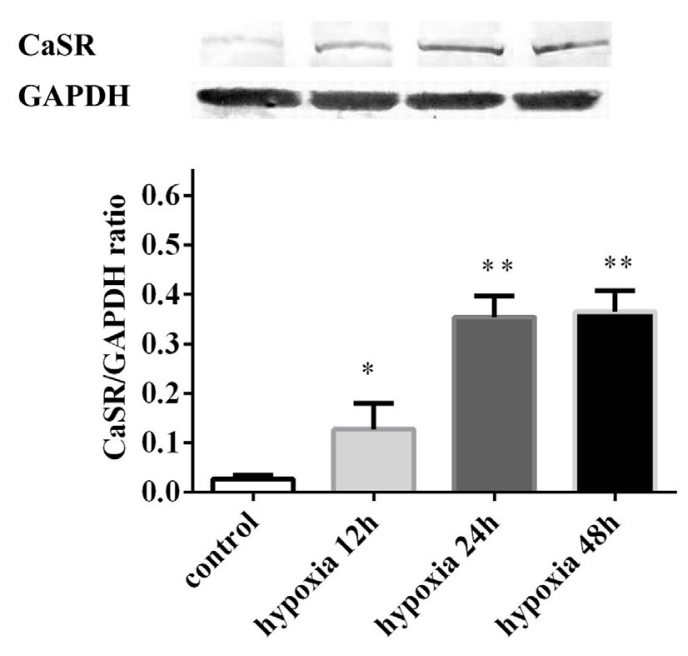

Figure 1: Hypoxia increases the expression of CaSR in human pulmonary arterial smooth muscle cells (HPASMCs). ${ }^{*} P<0.05$ versus control group, ${ }^{* *} P<0.01$ versus control group.
Hypoxia increases proliferation and promotes the phenotypic modulation in cultured HPASMCs

Moreover, we detect the proliferation assays and phenotype marker proteins of cultured in HPASMCs. The BrdU incorporation showed that the HPASMCs proliferation were increased demonstrably from $24 \mathrm{~h}$ under hypoxia when compared with the normoxic controls $(P<0.05$, Figure $2 \mathrm{~A}$ ), and western blot analysis revealed that the expression of PCNA and Ki67 were increased from $12 \mathrm{~h}$ and peaked at $24 \mathrm{~h}(P<0.05$, Figure $2 \mathrm{C}-\mathrm{D})$. Afterwards, the results of cell cycle analysis showed that proportion of cells from $\mathrm{G} 1$ phase to $\mathrm{S}$ phase was increased under hypoxia $(P<0.05$, Figure $2 \mathrm{~B})$.

Meanwhile, we found that the OPN expression was obviously increased, while the expressions of SMA $\alpha$ and calponin were markedly decreased in HPASMCs from 12 $\mathrm{h}$ under hypoxia when compared with the control group $(P$ $<0.05$; Figure 3). These data showed that the proliferation, cell cycle progression, and phenotypic modulation (from contractile phenotype to synthetic phenotype) of HPASMCs were induced under hypoxic conditions.

\section{The increasing proliferation of HPASMCS and promotion of phenotypic modulation is associated with the activation of autophagy induced by hypoxia}

To verify whether autophagy was related to the process that hypoxia induced proliferation of HPASMCs, cells were incubated under hypoxia for different times $(12$, 24 , and $48 \mathrm{~h}$ ). The expression of autophagic protein was observed by western blot analysis. The expression of LC3-II was increased markedly from $12 \mathrm{~h}$ (Figure 4). The results showed that the level of autophagy was increased in the early stage of hypoxia in a time-dependent manner.

To demonstrate the function of autophagy in HPASMCs and phenotypic modulation activated by hypoxia, a selective autophagy inhibitor, 3-MA, was added into hypoxic cell models. The inhibitor has no obvious toxic effects for certain cells including HPASMCs. ${ }^{[1]}$ When compared with the hypoxia $24 \mathrm{~h}$ group, the group in which 3-MA was added showed reduced expression of LC3-II, which indicate that 3-MA control the autophagy activated under hypoxia (Figure 5A). However, there was no variation in the expression of CaSR when 3-MA was given under hypoxia (Figure 5B). In addition, these data showed that the addition of 3-MA reduced the proliferation of HPASMC and reversed the phenotypic modulation of HPASMCs from synthetic phenotype to contractile phenotype at hypoxia for $24 \mathrm{~h}$ (Figure $5 \mathrm{C}-\mathrm{F}$ ), which indicate that the activation of autophagy maybe necessary for HPASMCs proliferation and phenotypic modulation under hypoxia, and CaSR is possibly the upstream factor of autophagy. 
A

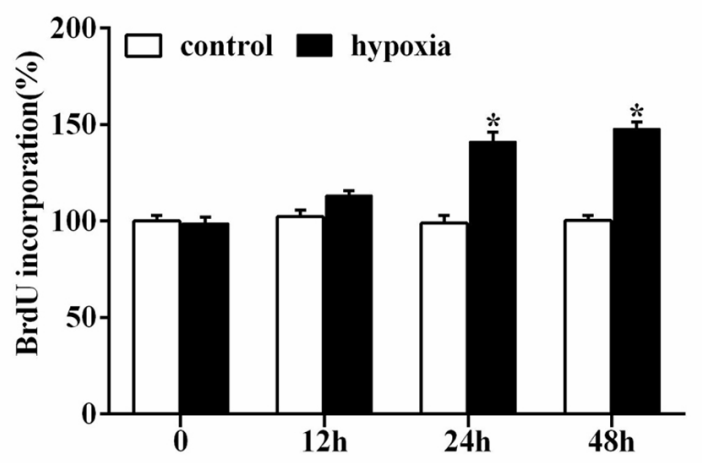

$\mathrm{B}$

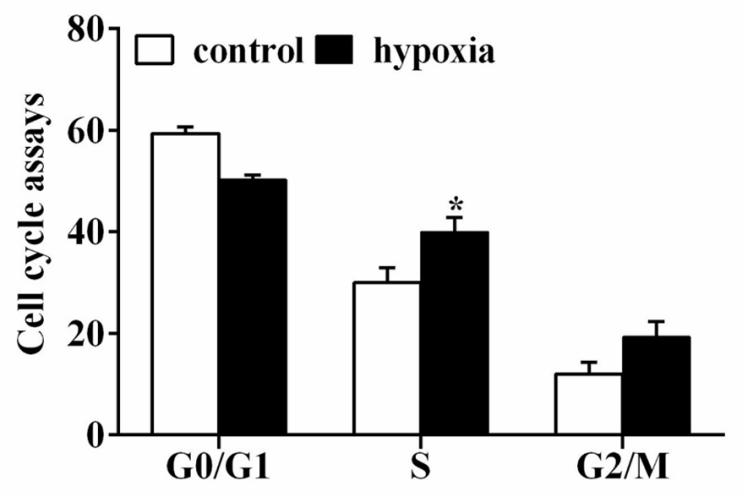

$\mathrm{C}$

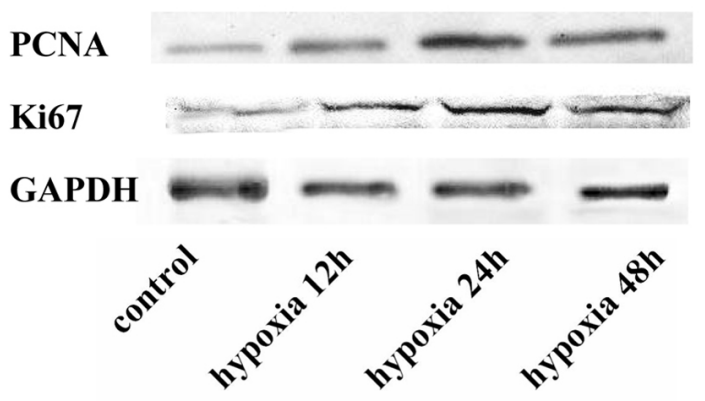

$\mathrm{D}$

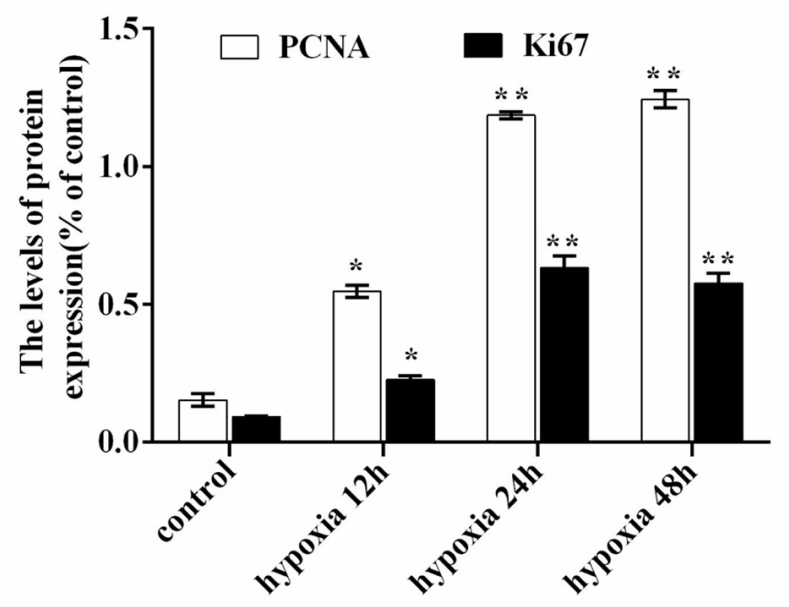

Figure 2: Hypoxia increases the proliferation and cell cycle progression of HPASMCs. (A) HPASMCs proliferation was measured by 5-bromo-2'-deoxyuridine (BrdU) incorporation. Data are presented as mean $\pm \mathrm{SEM}, n=5 .{ }^{*} P<0.05$ versus normoxia group. (B) Cell cycle analysis of HPASMCs in hypoxia condition at $24 \mathrm{~h}$ by flow cytometry. The results were expressed as relative cell growth in percentage, which was compared with a $21 \%$ oxygen control group. The concentration of $21 \%$ oxygen was set as control. $n=5$ for each group. ${ }^{*} P<0.05$ versus normoxia group. (C) Expression of PCNA and Ki67 proteins in HPASMCs under hypoxia. (D) Densitometry was applied to quantify PCNA and Ki67 protein density. Data were presented as mean \pm SEM, $n=5$. ${ }^{*} P<0.05$ versus control group, ${ }^{* *} P<0.01$ versus control group

A

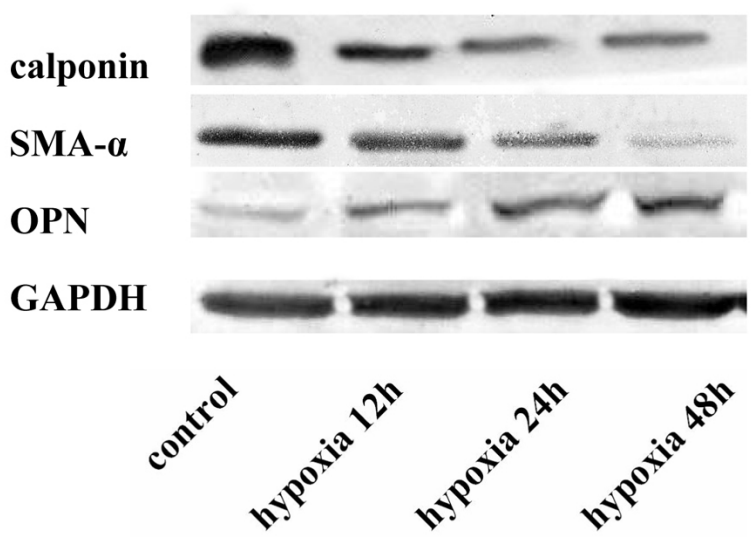

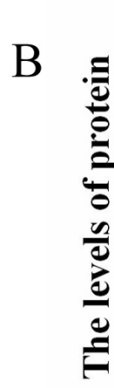

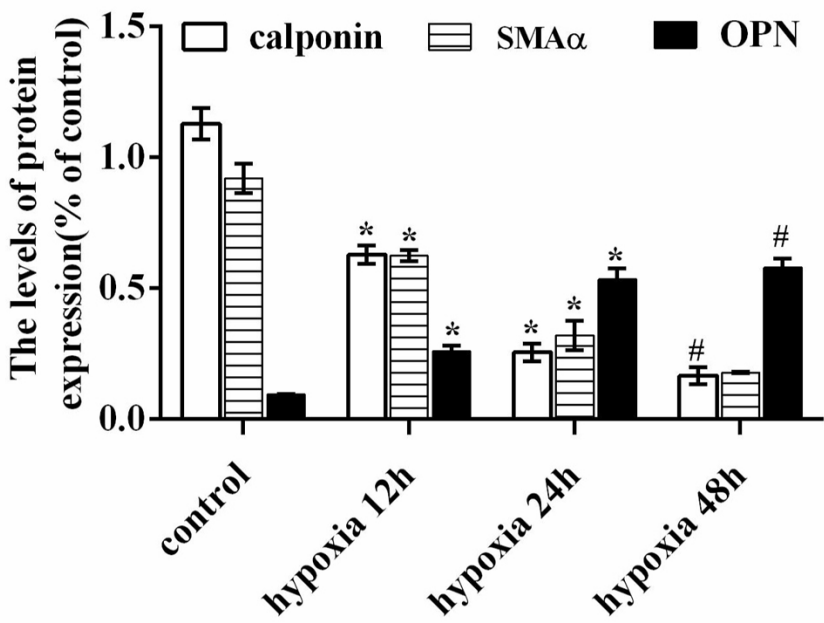

Figure 3: Hypoxia stimulates the phenotypic modulation of HPASMCs. (A) Phenotype marker proteins expression in HPASMCs detected by western blot. (B) Densitometric analysis of (A). Data were presented as mean \pm SEM. ${ }^{*} P<0.05$ versus control; ${ }^{*} P<0.05$ versus hypoxia for $24 \mathrm{~h}$. 

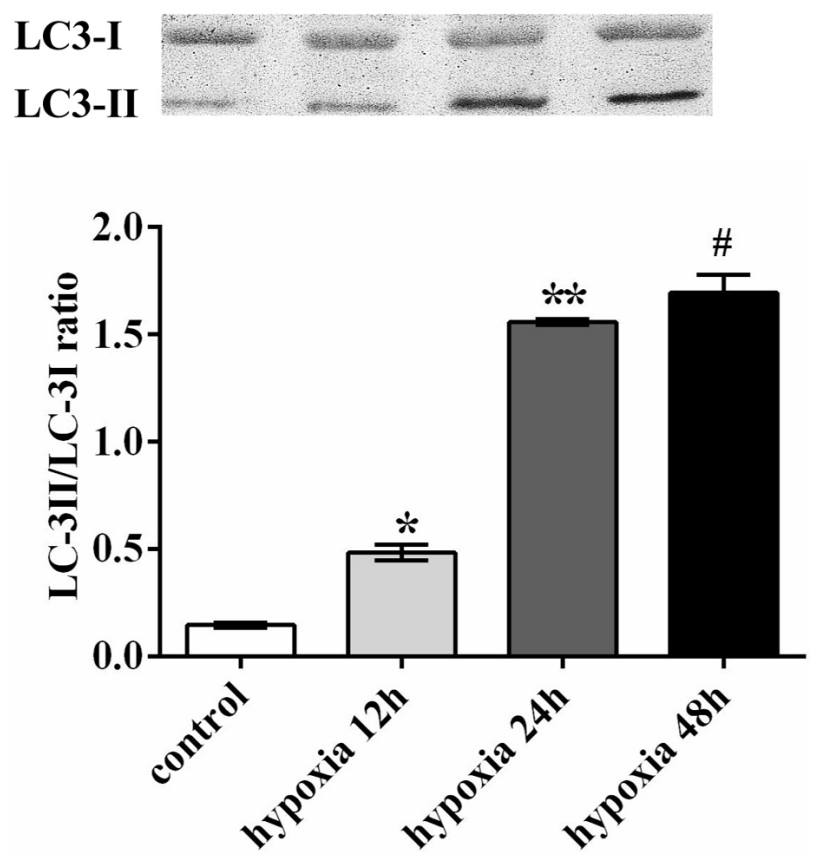

Figure 4: Activation of autophagy in HPASMCs under hypoxia. Data are presented as mean \pm SEM. ${ }^{*} P<0.05$ versus control; ${ }^{*} P<0.01$ versus control group; ${ }^{\#} P<0.05$ versus hypoxia for 24

\section{NPS2390 decreases proliferation and reverses phenotypic modulation via inhibiting autophagy in PASMCs under hypoxia}

To identify whether the function of CaSR is involved with the regulation of autophagy in HPASMCs proliferation and phenotypic modulation under hypoxia, HPASMCs were treated with NPS2390 $(10 \mu \mathrm{M}$, CaSR inhibitor $)$ for $24 \mathrm{~h}$ under hypoxic or normoxic conditions. Our results showed that the addition of NPS2390 reduced the proliferation and reversed phenotypic modulation in HPASMCs under hypoxia (Figure 6A and B). Meanwhile, the expression of LC3-II was also decreased after treating with NPS2390 (Figure 6C). The results indicate that the inhibition of CaSR suppressed the autophagy of HPASMCs under hypoxia (Figure 6D).

\section{Changes in $\left[\mathrm{Ca}^{2+}\right]_{i}$ in different groups}

We found that hypoxia markedly enhanced $\left[\mathrm{Ca}^{2+}\right]_{\mathrm{i}}$, and the addition of $\mathrm{R} 568(30 \mu \mathrm{M})$ raised a cooperativity as the $\left[\mathrm{Ca}^{2+}\right]_{\mathrm{i}}$ further increases in the hypoxia $+\mathrm{R} 568$ group $(P<0.05)$ when compared with the hypoxia group. But NPS2390 can suppress these changes (Figure 7).

\section{NPS2390 inhibits PI3K PI3K/Akt/mTOR pathways in HPASMCs under hypoxia}

Next, we intend to identify whether the decreasing level of autophagy activated by NPS2390 was attributed to the change in PI3K/Akt/mTOR pathways. After the addition of NPS2390 for $24 \mathrm{~h}$ under hypoxia, the expressions of p-PI3K, p-Akt, and p-mTOR were increased (Figure $8 \mathrm{~A}$ and $\mathrm{B}$ ). To further verify whether the function of NPS2390 is dependent on the PI3K/Akt pathways, the PI3K inhibitor LY294002 $(10 \mu \mathrm{M})$ was added together with the CaSR agonist R568 into HPASMCs under hypoxia. LY294002 suppressed the activation of Akt and its downstream mTOR, when compared with the R568 treatment hypoxia group. Furthermore, the function of NPS2390 on autophagy-associated protein was verified by western blot. The expression of LC3-II was upregulated by the addition of R568 at hypoxia for $24 \mathrm{~h}$ when compared with the hypoxic group without any treatment (Figure 8C and D). The LY294002 treatment obviously controls the expression of LC3-II when compared to the R568 treatment hypoxia group (Figure 8E).

These results demonstrated that the PI3K/Akt signaling pathway targets autophagy activation that is dependent on mTOR inhibition, which may be related to promote the suppression of HPASMC proliferation by NPS2390.

\section{DISCUSSION}

Our study identifies a novel function for CaSR in controlling autophagy in PASMCs. We observed that NPS2390, CaSR antagonists, significantly inhibit hypoxia-induced autophagy. Meanwhile, addition of autophagy inhibitor not only markedly suppressed the hyperproliferation of PASMCs under hypoxia but also reversed the phenotypic modulation of PASMCs from the contractile phenotype to the synthetic phenotype stimulate by hypoxia. These findings confirm that NPS2390 can inhibit hypoxia-induced pulmonary vascular remodeling by regulating autophagy to inhibit proliferation and phenotypic modulation of PASMCs.

Pulmonary hypertension is a serious and fatal circulatory disease, mainly caused by excessive proliferation of vascular wall cells, eventually occluding small pulmonary artery. ${ }^{[12-14]}$ Although several studies have made some progress in the treatment of pulmonary hypertension in the recent years, the current treatment methods are not satisfactory. ${ }^{[15]}$ Therefore, a better understanding of the pathogenesis of PAH and the selection of more effective treatments are imminent. In 2015, hypoxia-induced pulmonary hypertension $(\mathrm{HPH})$ was listed as one of the most common types in PAH by the European Society of Cardiology and Respiratory Society. ${ }^{[16]}$ The pathological basis that occurs in $\mathrm{HPH}$ contains hypoxic pulmonary vasoconstriction and hypoxic pulmonary vascular remodeling. ${ }^{[17,18]}$ Moreover, studies have observed that over proliferation of PASMCs plays an important role in the pathology of hypoxic pulmonary vascular remodeling. ${ }^{[19,20]}$ Meanwhile, PASMCs 
A LC3-I LC3-II

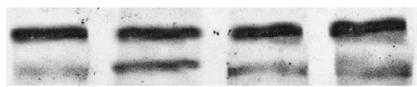

$\mathrm{C}$
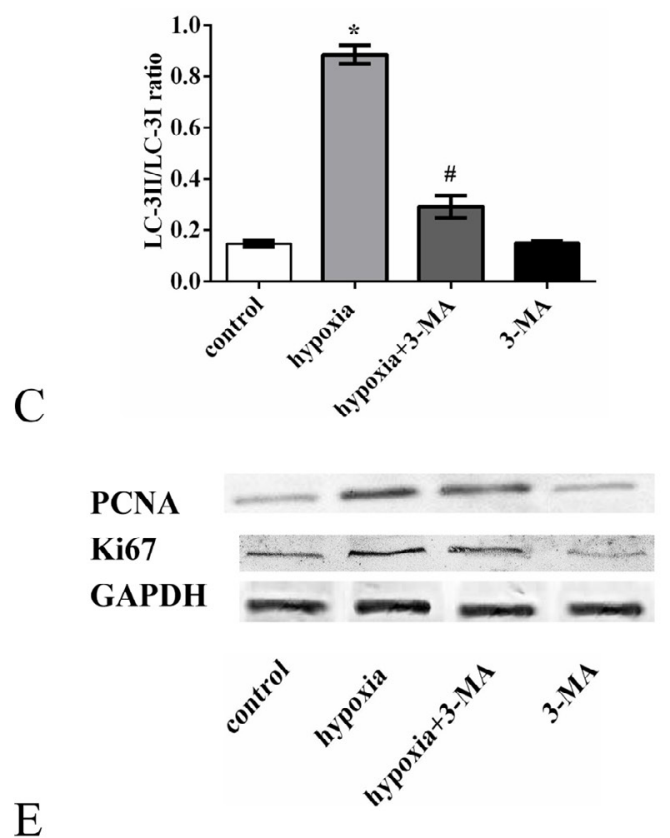

$\mathrm{E}$

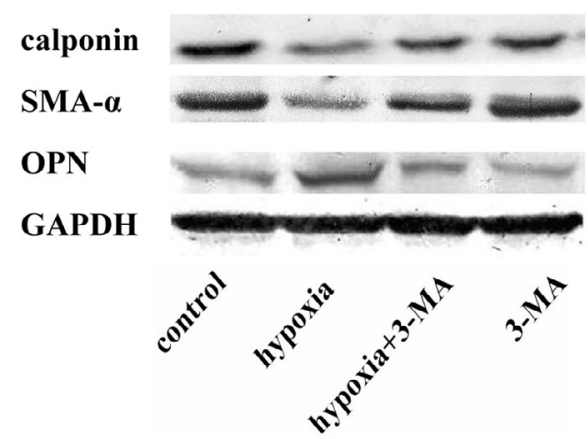

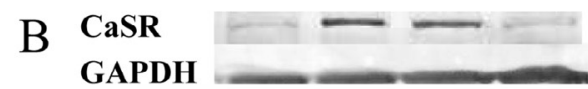

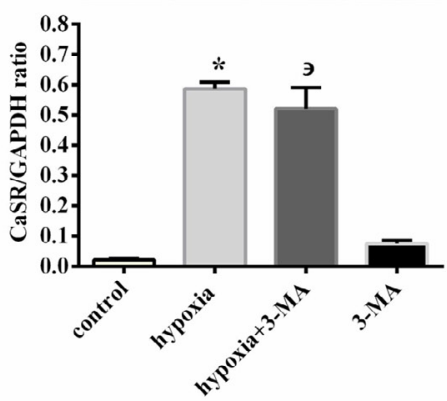

$\mathrm{D}$
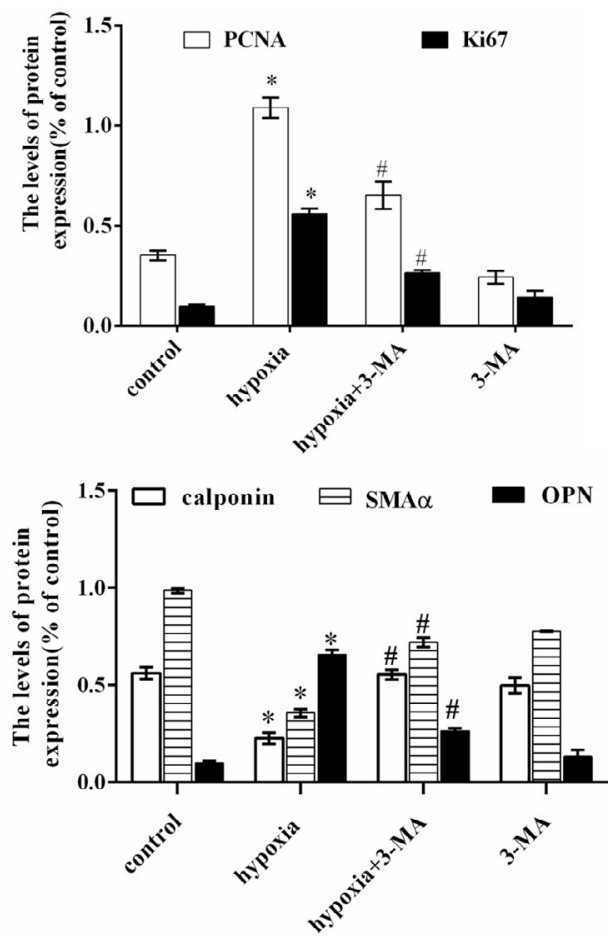

Figure 5: 3-MA inhibits autophagy and CaSR expression, decreases the proliferation, and reverses the phenotypic modulation of HPASMCs induced by hypoxia. (A) The levels of LC3-II and LC3-I were measured in the HPASMCs under hypoxia by western blot analysis. (B) The levels of CaSR were measured in the HPASMCs under hypoxia by western blot analysis. (C) The levels of PCNA and Ki67 were measured in the HPASMCs under hypoxia by western blot analysis. (D) Densitometric analysis of (C). (E) The levels of phenotype marker protein were measured in the HPASMCs under hypoxia by western blot analysis. (F) Densitometric analysis of (E). Data were presented as mean \pm SEM, $n=5$. ${ }^{*} P<0.05$ versus control group, ${ }^{\#} P<0.05$ versus hypoxia group, ${ }^{\supset} P>0.05$ versus hypoxia group

phenotypic modulation is characterized by an increase in smooth-muscle-cell-specific expression of phenotypeassociated proteins that contribute to the proliferation of PASMCs and vascular remodeling of pulmonary hypertension. Although the study of pathological changes in HPH has become clearer, the cellular mechanisms related to excessive and uncontrolled growth of vascular wall cells remain unclear.

An increase in the concentration of cytosolic calcium $\left(\left[\mathrm{Ca}^{2+}\right]_{\mathrm{cyt}}\right)$ in PASMCs is the main cause for pulmonary vasoconstriction and a critical stimulation for PASMCs proliferation and migration. ${ }^{[21]}$ As the major regulator of intracellular calcium concentrations, the CaSR can respond to the changes in extracellular calcium concentrations $\left(\left[\mathrm{Ca}^{2+}\right]\right)$ and its activation can induce the release of calcium from the sarcoplasmic reticulum. ${ }^{[22,23]}$ Our previous study have verified that CaSR activation is related to hypoxiainduced proliferation of rat PASMCs via MEK1/ERK1,2 and PI3K signal pathways. Recently, we also discovered that the expression of CaSR was increased and involved the phenotypic modulation of PASMCs in small pulmonary artery and vascular remodeling in hypoxic rat. In our study, we used human PASMCs to further observe the underlying 
A

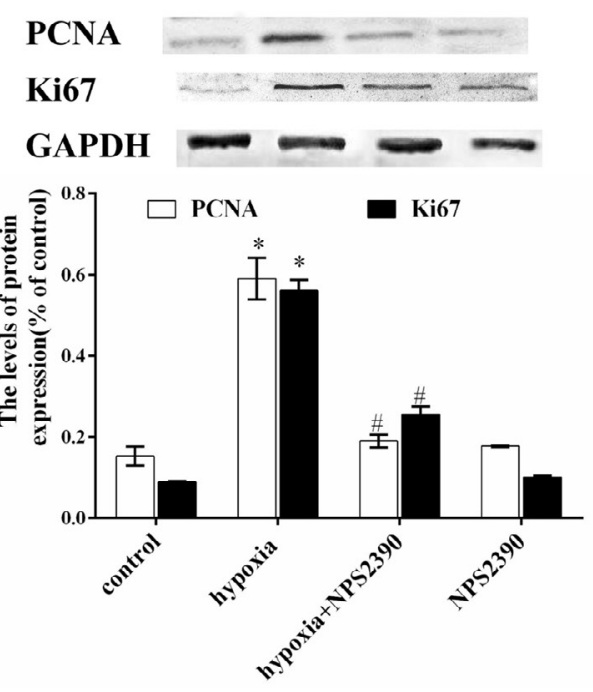

$\mathrm{C}$
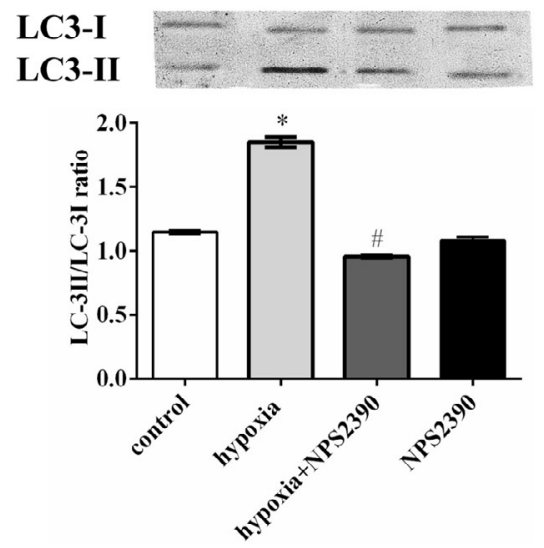

$\mathrm{B}$
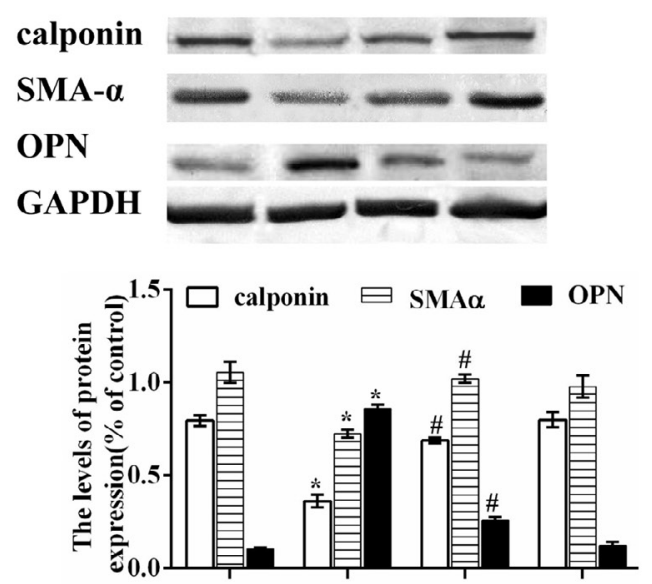

$\mathrm{D}$
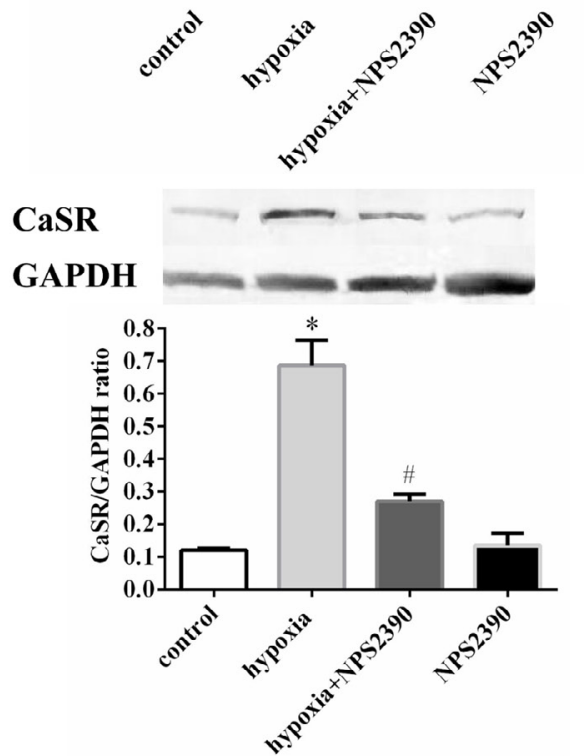

Figure 6: NPS2390 inhibits autophagy and CaSR expression, decreases the proliferation, and reverses the phenotypic modulation of HPASMCs induced by hypoxia. (A) The levels of PCNA and Ki67 were measured in the HPASMCs under hypoxia by western blot analysis. (B) The levels of phenotype marker protein were measured in the HPASMCs under hypoxia by western blot analysis. (C) The levels of LC3-II and LC3-I were measured in the HPASMCs under hypoxia by western blot analysis. (D) The levels of CaSR were measured in the HPASMCs under hypoxia by western blot analysis. Data were presented as mean \pm SEM, $n=5$. ${ }^{*} P<0.05$ versus control group, ${ }^{\#} P<0.05$ versus hypoxia group.

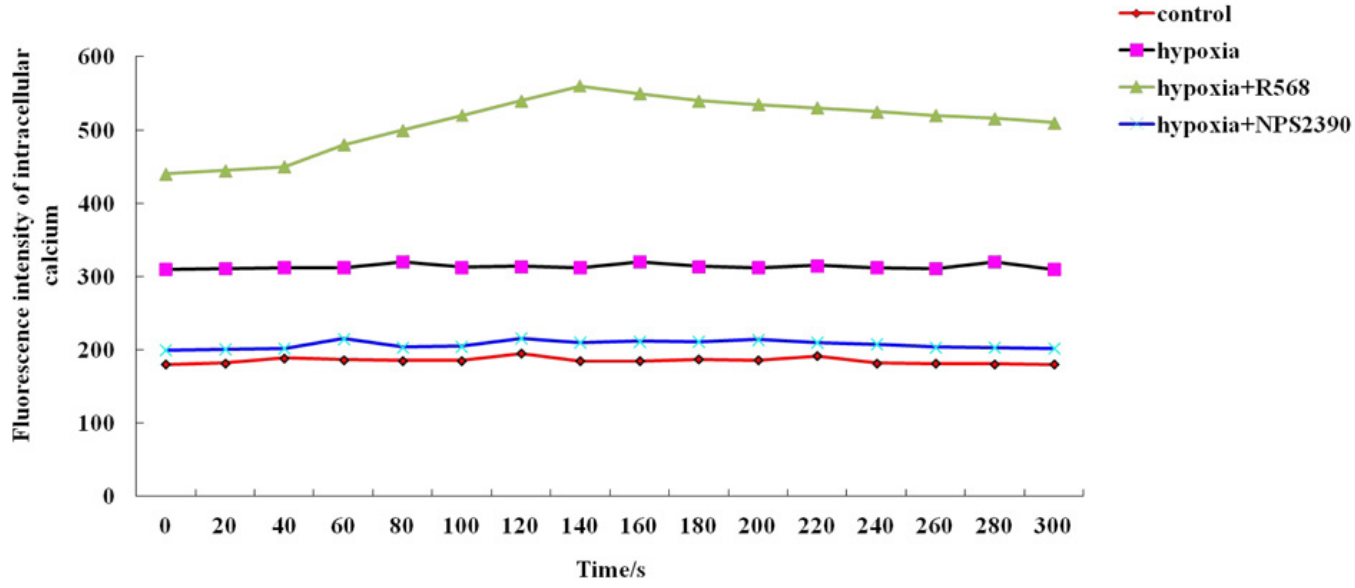

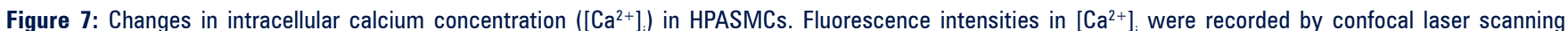
microscopy in different treatments. Compared with the control group, hypoxia significantly increased $\left[\mathrm{Ca}^{2+}\right]_{i^{\prime}}$, and this increase was enhanced by $\mathrm{R} 568$ $(P<0.05$ versus hypoxia group) but was attenuated by the addition of NPS2390 $(P<0.05$ versus hypoxia group). 
A

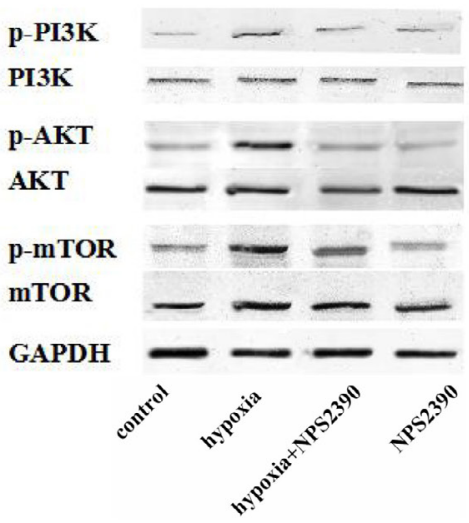

C

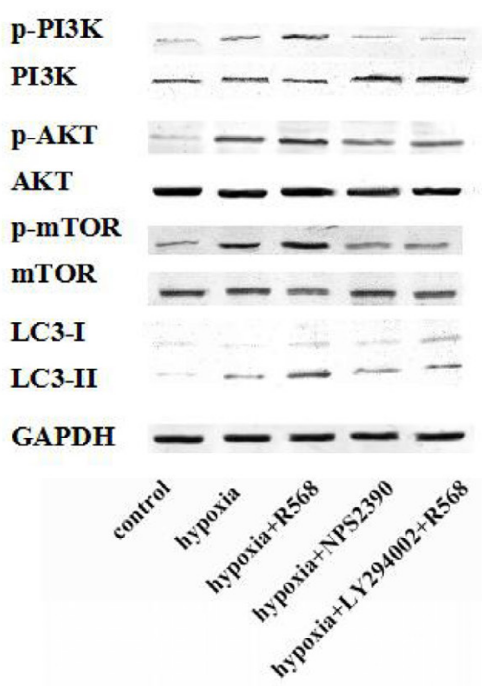

B

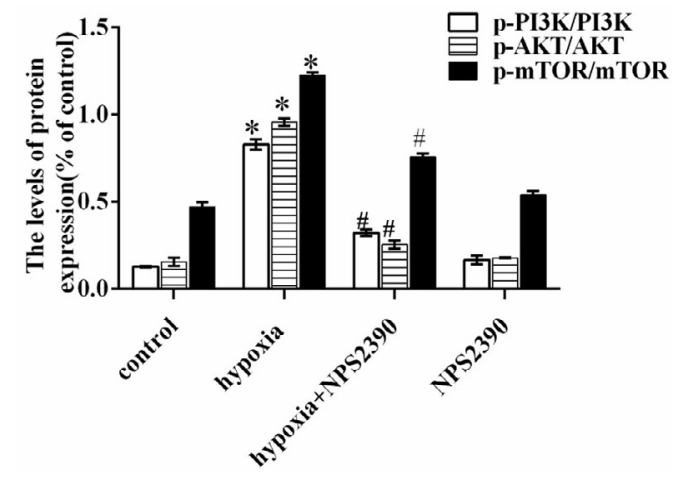

D

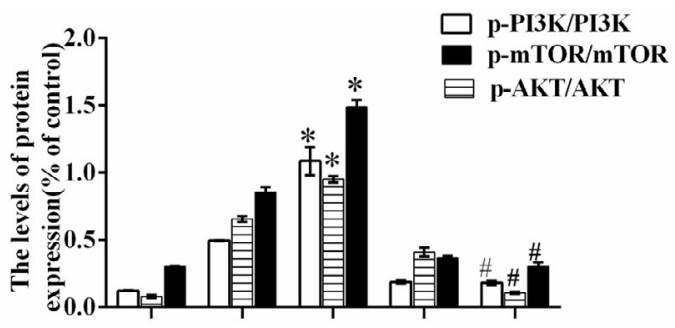

$\mathrm{E}$

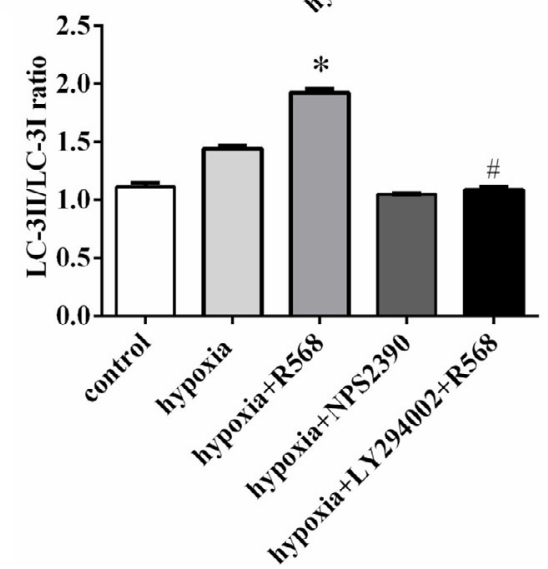

Figure 8: The effect of NPS2390 on autophagy in HPASMCs induced by hypoxia is related to the regulation of PI3K/Akt/mTOR pathways. (A) NPS2390 decreases the phosphorylation of PI3K/Akt/mTOR signals. The protein expressions were measured by western blot analysis. (B) Densitometry was applied to quantify the protein density; data were presented as mean $\pm \mathrm{SEM}, n=5 .{ }^{*} P<0.05$ versus control group, ${ }^{*} P<0.05$ versus hypoxia group. (C) Expression of phosphorylated-Pl3K/Akt/ mTOR and LC3 proteins in HPASMCs under hypoxia with NPS2390, R568, and Akt inhibitor LY294002. (D) The ratio of normalized LC3-II to LC3-I. (E) Densitometry was applied to quantify the density of phosphorylated-PI3K/AKT/mTOR protein; data were presented as mean $\pm \mathrm{SEM}, n=5 .{ }^{*} P<0.05$ versus hypoxia group, ${ }^{\#} P<0.05$ versus R568-treated hypoxia group.

function of the CaSR in pulmonary vascular remodeling induced under hypoxia; we found the same changes in the expression of CaSR and the proliferation factors PCNA and Ki67. Meanwhile, we also observed that hypoxia induced the increased expression of synthetic phenotype marker protein osteopontin and decreased the expression of contractile phenotype marker proteins SMA- $\alpha$ and calponin. CaSR antagonist NPS 2390 weakened the effect of hypoxia. The results showed that hypoxia can induce increased expression of CaSR and proliferation and phenotypic modulation of HPASMCs. Therefore, we hypothesized that hypoxia-induced CaSR activation plays an important role in promoting PASMCs proliferation and phenotypic modulation, and this change is due to CaSR-mediated increase in intracellular calcium concentration. 
To verify the hypothesis, we detected the level of $\left[\mathrm{Ca}^{2+}\right]_{\mathrm{i}}$ and observed that lower oxygen conditions obviously increased $\left[\mathrm{Ca}^{2+}\right]_{\mathrm{i}}$, this increase can be further upregulated by CaSR agonist R568 and downregulated by NPS2390. NPS R568 is an allosteric agonist of CaSR, also known as calcimimetic, which binds specifically to CaSR, thereby altering its spatial conformation and increasing the sensitivity of CaSR to extracellular $\mathrm{Ca}^{2+} .{ }^{[24]}$ Moreover, autophagy, as an important process of cell death and survival, has been shown to be involved in mediating SMC proliferation. ${ }^{[25,26]}$ However, whether $\mathrm{CaSR}$ regulates phenotypic modulation of PASMCs via autophagy remain elusive.

Autophagy is a highly conserved protein or organelle degradation process found in eukaryotes ${ }^{[2]}$ and is related to the pathogenesis of HPAH..$^{[4]}$ So far, only a few sporadic studies of autophagy have been published. Seon-Jin Lee have found that autophagic protein LC3B play a protective role during the development of $\mathrm{PAH}$ through regulating hypoxic-induced cell proliferation. ${ }^{[4]}$ Recently, studies have found that chloroquine inhibits autophagy and blocks lysosomal degradation of bone morphogenetic protein type II receptors, thereby inhibiting the proliferation and increased apoptosis of PASMCs in HPH animal and cell models. ${ }^{[28]}$ The latest study shows that the activation of autophagy is related to hypoxia-induced proliferation and migration of PASMCs and the inhibition of autophagy leads to decreased cell proliferation and cell cycle arrest, ${ }^{[29]}$ which is consistent with our results.

In this study, we showed that hypoxia-induced activation of autophagy is related to the proliferation and phenotypic modulation of PASMCs, and autophagy-specific inhibitor, 3-MA, can reduce proliferation and reverse the phenotypic modulation of PASMCs, proposing that the activation of autophagy converted the PASMCs from contractile phenotype to synthetic phenotype and promoted PASMCs proliferation under hypoxic condition, which may be an important factor in the progression of $\mathrm{HPH}$ disease and vascular remodeling. Concurrently, CaSR antagonist, NPS2390, inhibits the autophagy induced by hypoxia. These results showed that CaSR antagonists treatment may suppress the proliferation and reverse phenotypic modulation of PASMCs through regulating autophagy level.

CaSR has been shown to promote the activation of the PI3K/Akt pathway, which plays a protective function in cell proliferation. Moreover, it is common knowledge that as an upstream pathway, the PI3K/Akt/mTOR signal is critical for the regulation of autophagy. ${ }^{[30-32]}$ But the function of $\mathrm{CaSR}$ and PI3K/Akt/mTOR in HPASMCs of hypoxic cell models has not been discussed. In the current study, we first found that the inhibition of the PI3K/Akt/mTOR pathway is critical for the inhibition of hypoxia-induced autophagy by NPS2390. In addition, NPS2390 reduces autophagy levels in PASMC by inhibiting the downstream signaling of Akt and mTOR. The data from these in vitro experiments suggest that regulation of autophagy and its downstream signaling may be related to the effects of NPS2390, which is beneficial for inhibiting vascular remodeling in $\mathrm{HPH}$.

However, our findings need further research and confirmation in vivo to confirm the role of NPS2390 in the regulation of autophagy involved in vascular remodeling and HPH formation by hypoxic animal models, further verifying the feasibility of NPS2390 as a therapeutic target for HPH.

In summary, our results demonstrated that CaSR inhibition regulates proliferation, cell cycle progression, and phenotypic modulation in PASMCs caused by hypoxia, which relies on PI3K/Akt/mTOR signal pathway. Inhibition of this pathway prevents PASMC proliferation and reverses HPVR. Our study reveals the mechanism of HPVR and provides more targets for the treatment of $\mathrm{PAH}$

\section{Source of Foundation}

The present study was supported by National Natural Science Foundation of China, grant No. $81270311,81300200,81270273$.

\section{Conflict of Interest}

The authors declare that they have no competing interests.

\section{REFERENCES}

1. Mizushima N, Komatsu M. Autophagy: renovation of cells and tissues. Cell 2011; 4: 728-41.

2. Klionsky DJ, Abdalla FC, Abeliovich H, Abraham RT, Acevedo-Arozena A, Adeli K, et al. Guidelines for the use and interpretation of assays for monitoring autophagy. Autophagy 2012; 4: 445-544.

3. Li H, Li J, Li Y, Singh P, Cao L, Xu LJ, et al. Sonic hedgehog promotes autophagy of vascular smooth muscle cells. Am J Physiol Heart Circ Physiol 2012; 11: H1319-31.

4. Lee SJ, Smith A, Guo L, Alastalo TP, Li M, Sawada H, et al. Autophagic protein LC3B confers resistance against hypoxia-induced pulmonary hypertension. Am J Respir Crit Care Med 2011; 5: 649-58.

5. Nussenzweig SC, Verma S, Finkel T. The role of autophagy in vascular biology. Circ Res 2015; 3: 480-8.

6. Garcia-Miguel M, Riquelme JA, Norambuena-Soto I, Morales PE, Sanhueza-Olivares F, Nunez-Soto C, et al. Autophagy mediates tumor necrosis factor-alpha-induced phenotype switching in vascular smooth muscle A7r5 cell line. PLoS One 2018; 5: e0197210.

7. Xie X, Man X, Zhu Z, Yuan D, Huang S. Tumor suppressor RIZ1 in obesity and the PI3K/AKT/mTOR pathway. Obesity (Silver Spring) 2016; 2: 389-97. 
8. Zhang HW, Hu JJ, Fu RQ, Liu X, Zhang YH, Li J, et al. Flavonoids inhibit cell proliferation and induce apoptosis and autophagy through downregulation of PI3Kgamma mediated PI3K/AKT/mTOR/p70S6K/ULK signaling pathway in human breast cancer cells. Sci Rep 2018; 1: 11255.

9. He C, Klionsky DJ. Regulation mechanisms and signaling pathways of autophagy. Annu Rev Genet 2009: 67-93.

10. Xie X, Zhu T, Chen L, Ding S, Chu H, Wang J, et al. MCPIP1-induced autophagy mediates ischemia/reperfusion injury in endothelial cells via HMGB1 and CaSR. Sci Rep. 2018; 1:1735.

11. Bao J, Shi Y, Tao M, Liu N, Zhuang S, Yuan W. Pharmacological inhibition of autophagy by 3-MA attenuates hyperuricemic nephropathy. Clin Sci (Lond). 2018; 21:2299-2322.

12. Humbert M, Sitbon O, Simonneau G. Treatment of pulmonary arterial hypertension. N Engl J Med 2004; 14: 1425-36.

13. Pietra GG, Capron F, Stewart S, Leone O, Humbert M, Robbins IM, et al. Pathologic assessment of vasculopathies in pulmonary hypertension. J Am Coll Cardiol 2004; 12 Suppl S: 25S-32S.

14. Wei H, Zhang D, Liu L, Xia W, Li F. Rho signaling pathway enhances proliferation of PASMCs by suppressing nuclear translocation of Smad1 in PAH. Exp Ther Med 2019; 1: 71-8.

15. Liu HL, Chen XY, Li JR, Su SW, Ding T, Shi CX, et al. Efficacy and Safety of Pulmonary Arterial Hypertension-specific Therapy in Pulmonary Arterial Hypertension: A Meta-analysis of Randomized Controlled Trials. Chest 2016; 2: 353-66.

16. Galie N, Humbert M, Vachiery JL, Gibbs S, Lang I, Torbicki A, et al. 2015 ESC/ERS Guidelines for the Diagnosis and Treatment of Pulmonary Hypertension. Rev Esp Cardiol (Engl Ed). 2016; 2:177.

17. Chan SY, Loscalzo J. Pathogenic mechanisms of pulmonary arterial hypertension. J Mol Cell Cardiol 2008; 1: 14-30.

18. Li X, He Y, Xu Y, Huang X, Liu J, Xie M, et al. KLF5 mediates vascular remodeling via HIF-1alpha in hypoxic pulmonary hypertension. Am J Physiol Lung Cell Mol Physiol 2016; 4: L299-310.

19. Stenmark KR, Fagan KA, Frid MG. Hypoxia-induced pulmonary vascular remodeling: cellular and molecular mechanisms. Circ Res 2006; 7: 675-91.

20. Mao SZ, Fan XF, Xue F, Chen R, Chen XY, Yuan GS, et al. Intermedin modulates hypoxic pulmonary vascular remodeling by inhibiting pulmonary artery smooth muscle cell proliferation. Pulm Pharmacol Ther 2014; 1: 1-9.

21. Tang H, Yamamura A, Yamamura H, Song S, Fraidenburg DR, Chen J, et al. Pathogenic role of calcium-sensing receptors in the development and progression of pulmonary hypertension. Am J Physiol Lung Cell Mol Physiol 2016; 9: L846-59.
22. Riccardi D. Parathyroid hormone-independent role for the calciumsensing receptor in the control of urinary calcium excretion. J Am Soc Nephrol 2012; 11: 1766-8.

23. Lu FH, Fu SB, Leng X, Zhang X, Dong S, Zhao YJ, et al. Role of the calcium-sensing receptor in cardiomyocyte apoptosis via the sarcoplasmic reticulum and mitochondrial death pathway in cardiac hypertrophy and heart failure. Cell Physiol Biochem 2013; 4-5: 728-43.

24. Rybczynska A, Boblewski K, Lehmann A, Orlewska C, Foks H. Pharmacological activity of calcimimetic NPS R-568 administered intravenously in rats: dose dependency. Pharmacol Rep 2006; 4: 533-9.

25. Patel AS, Morse D, Choi AM. Regulation and functional significance of autophagy in respiratory cell biology and disease. Am J Respir Cell Mol Biol 2013; 1: 1-9.

26. Wu H, Song A, Hu W, Dai M. The Anti-atherosclerotic Effect of Paeonol against Vascular Smooth Muscle Cell Proliferation by Up-regulation of Autophagy via the AMPK/mTOR Signaling Pathway. Front Pharmacol 2017: 948.

27. Yang Z, Klionsky DJ. Eaten alive: a history of macroautophagy. Nat Cell Biol 2010; 9: 814-22.

28. Long L, Yang X, Southwood M, Lu J, Marciniak SJ, Dunmore BJ, et al. Chloroquine prevents progression of experimental pulmonary hypertension via inhibition of autophagy and lysosomal bone morphogenetic protein type II receptor degradation. Circ Res 2013; 8: 1159-70.

29. Zhang H, Gong $Y$, Wang Z, Jiang L, Chen R, Fan X, et al. Apelin inhibits the proliferation and migration of rat PASMCs via the activation of PI3K/ Akt/mTOR signal and the inhibition of autophagy under hypoxia. J Cell Mol Med 2014; 3: 542-53.

30. Liu M, Zhao G, Zhang D, An W, Lai H, Li X, et al. Active fraction of clove induces apoptosis via PI3K/Akt/mTOR-mediated autophagy in human colorectal cancer HCT-116 cells. Int J Oncol 2018; 3: 1363-73.

31. Wang B, Zhong Y, Li Q, Cui L, Huang G. Autophagy of macrophages is regulated by PI3k/Akt/mTOR signalling in the development of diabetic encephalopathy. Aging (Albany NY) 2018; 10: 2772-82.

32. Yang J, Pi C, Wang G. Inhibition of PI3K/Akt/mTOR pathway by apigenin induces apoptosis and autophagy in hepatocellular carcinoma cells. Biomed Pharmacother 2018: 699-707.

How to cite this article: R Peng X, Wei C, Li HZ, Li HX, Bai SZ, Wang LN, et al. NPS2390, a selective calcium-sensing receptor antagonist controls the phenotypic modulation of hypoxic human pulmonary arterial smooth muscle cells by regulating autophagy. J Transl Int Med 2019; 7: 59-68. 